Dislocation Multi-junctions and Strain Hardening

Vasily Bulatov, Luke Hsiung, Meijie Tang, Athanasios Arsenlis, Maria Bartelt, We Cai, Jeff Florando, Masato Hiratani, Moon Rhee, Gregg Hommes, Tim Pierce, Tomas Diaz de la Rubia

June 21, 2006

Nature 
This document was prepared as an account of work sponsored by an agency of the United States Government. Neither the United States Government nor the University of California nor any of their employees, makes any warranty, express or implied, or assumes any legal liability or responsibility for the accuracy, completeness, or usefulness of any information, apparatus, product, or process disclosed, or represents that its use would not infringe privately owned rights. Reference herein to any specific commercial product, process, or service by trade name, trademark, manufacturer, or otherwise, does not necessarily constitute or imply its endorsement, recommendation, or favoring by the United States Government or the University of California. The views and opinions of authors expressed herein do not necessarily state or reflect those of the United States Government or the University of California, and shall not be used for advertising or product endorsement purposes. 


\section{Dislocation multi-junctions and strain hardening}

Vasily V. Bulatov*, Luke L. Hsiung*, Meijie Tang*, Athanasios Arsenlis*, Maria C. Bartelt*, Wei Cai*†, Jeff N. Florando*, Masato Hiratani*, Moon Rhee*, Gregg Hommes*, Tim G. Pierce*, Tomas Diaz de la Rubia*

*Lawrence Livermore National Laboratory, University of California, Livermore, California 94550, USA

$\dagger$ †epartment of Mechanical Engineering, Stanford University, Stanford, California 94305, USA

At the microscopic scale, the strength of a crystal derives from the motion, multiplication and interaction of distinctive line defects - dislocations. First theorized in $1934^{1-3}$ to explain low magnitudes of crystal strength observed experimentally, the existence of dislocations was confirmed only two decades later ${ }^{4,5}$. Much of the research in dislocation physics has since focused on dislocation interactions and their role in strain hardening: a common phenomenon in which continued deformation increases a crystal's strength. The existing theory relates strain hardening to pair-wise dislocation reactions in which two intersecting dislocations form junctions tying dislocations together, ${ }^{6,7}$. Here we report that interactions among three dislocations result in the formation of unusual elements of dislocation network topology, termed hereafter multi-junctions. The existence of multi-junctions is first predicted by Dislocation Dynamics (DD) and atomistic simulations and then confirmed by the transmission electron microscopy (TEM) experiments in single crystal molybdenum. In large-scale Dislocation Dynamics simulations, multi-junctions present very strong, nearly indestructible, obstacles to 
dislocation motion and furnish new sources for dislocation multiplication thereby playing an essential role in the evolution of dislocation microstructure and strength of deforming crystals ${ }^{8}$. Simulation analyses conclude that multi-junctions are responsible for the strong orientation dependence of strain hardening in $\mathrm{BCC}$ crystals.

The amount of slip produced by a propagating dislocation is quantified by its Burgers vector $\mathbf{b}$ equal to one of the (typically smallest) repeat vectors of the crystal lattice. What exactly happens when two dislocations collide depends on the lengths of two lines, their collision geometry and applied stress. Most significantly, the collision outcomes are affected by the Burgers vectors of two colliding dislocations. Given that a dislocation's energy is proportional to the square of its Burgers vector, the approximate Frank energy criterion ${ }^{9}$ predicts that, when $\left(\mathbf{b}_{1}+\mathbf{b}_{2}\right)^{2}<\mathbf{b}_{1}{ }^{2}+\mathbf{b}_{2}{ }^{2}$ or, equivalently, $\mathbf{b}_{1} \cdot \mathbf{b}_{2}<$ 0, the two lines will attract and merge into a product dislocation - junction - with Burgers vector $\mathbf{b}_{\mathrm{j}}=\mathbf{b}_{1}+\mathbf{b}_{2}$ thereby reducing the internal energy of the system. In particular, when $\mathbf{b}_{2}=-\mathbf{b}_{1}$, the two dislocations can annihilate completely leaving no product. Fig. 1(a,b) shows a junction-forming collision of two dislocation lines in a Dislocation Dynamics (DD) simulation (see the Methods section and Supplementary Discussion 1 for more details). The initial configuration consists of two straight dislocation lines of equal length made to intersect at their midpoints while their endpoints are rigidly fixed (Fig. 1(a)). Expressed in the units of the lattice constant, the Burgers vectors of two lines are $\mathbf{b}_{1}=$ $1 / 2[\overline{1} 11]$ and $\mathbf{b}_{2}=1 / 2[1 \overline{1} 1]$, typical of the body-centred-cubic (BCC) crystals. In the DD simulation, the elastic interaction between two lines causes them to merge into a junction dislocation with Burgers vector $\mathbf{b}_{\mathrm{j}}=\mathbf{b}_{1}+\mathbf{b}_{2}=$ [001] (Fig. 1(b), see also Supplementary Video 1). Owing to their fixed ends, the lines merge only partially (when $\mathbf{b}_{2}=-\mathbf{b}_{1}$ the lines will partially annihilate). Bounded at its ends by two triple nodes, the resulting junction zips along [111] direction because the two parent dislocations are allowed to 
move only on their individual glide planes (plane containing their Burgers vector $\mathbf{b}$ and initial line direction $\xi$ ) with normal vectors $\mathbf{n}_{1}=(01 \overline{1})$ and $\mathbf{n}_{2}=(10 \overline{1})$, respectively. Since most of the interacting dislocations move on non-parallel glide planes, attractive collisions zip junctions of limited length along the intersection lines of the glide planes. The frequency and strength of such pair-wise dislocation reactions tying dislocations together are believed to control the physics of strain hardening: a common phenomenon in which continued deformation increases a crystal's strength.

The existence and the important role of dislocations junctions in strain hardening has been confirmed by numerous theoretical ${ }^{6,7}$ and experimental ${ }^{10,11}$ studies and, more recently, by DD simulations ${ }^{12,13}$. Very recently, analyzing previously published ${ }^{14}$ and our own new simulations, we observed formation of complex topological connections in which more than two dislocation lines merge together. Curious about possible causes for such anomalous formations, we proceeded to investigate if attractive reactions among three or more dislocations are possible. For BCC crystals, one such candidate reaction is

$1 / 2[\overline{1} 11]+1 / 2[1 \overline{1} 1]+1 / 2[11 \overline{1}]=1 / 2[111]$.

$$
\begin{array}{llll}
\mathbf{b}_{1} & \mathbf{b}_{2} & \mathbf{b}_{3} & \mathbf{b}_{4}
\end{array}
$$

Considering that the elastic energy stored in a dislocation's strain field is $\propto\|\mathbf{b}\|^{2}$, the Frank estimate of the energy reduction in the above reaction, $\left(\left\|\mathbf{b}_{1}\right\|^{2}+\left\|\mathbf{b}_{2}\right\|^{2}+\left\|\mathbf{b}_{3}\right\|^{2}\right) /$ $\left\|\mathbf{b}_{4}\right\|^{2}=3$, is much greater than in the binary reaction shown in Fig. 1(b), $\left(\left\|\mathbf{b}_{1}\right\|^{2}+\left\|\mathbf{b}_{2}\right\|^{2}\right) /$ $\left\|\mathbf{b}_{\mathrm{j}}\right\|^{2}=1.5$. To see if such a reaction is indeed feasible, let us now overlay on top of the binary junction a third dislocation (Fig. 1(c)) with Burgers vector $\mathbf{b}_{3}=1 / 2[11 \overline{1}]$ and glide plane $\mathbf{n}_{3}=(1 \overline{1} 0)$. The DD simulation result is shown in Fig. 1(d), where the third dislocation with Burgers vector $\mathbf{b}_{3}$ has reacted with the junction dislocation and transformed it into a $1 / 2[111]$ dislocation, exactly in accord with proposed reaction (1) 
(Supplementary Video 2). The transformed junction segment with Burgers vector $\mathbf{b}_{4}$ of $1 / 2<111>$-type connects together three dislocations at its ends and is defined as a multijunction. Remarkably, the resulting multi-junction extends well beyond the original length of the binary junction, corroborating our expectation that this ternary reaction (1) can result in a much greater energy reduction than the classical binary reaction.

The nodes at each end of the multi-junction that tie together four dislocation lines we term multi-nodes or 4-nodes. These 4-nodes are distinctly different from simple crosses of two dislocations in that all four lines entering the node have different Burgers vectors. These 4-nodes are beautifully symmetric: all four distinct Burgers vectors of $1 / 2<111>$-type enter the 4-node exactly once. Therefore, it is the only possible 4-node of this kind in BCC crystals. Curiously, the same symmetric 4-node can be formed through four different reactions among three lines, for example $\mathbf{b}_{1}+\mathbf{b}_{2}+\left(-\mathbf{b}_{4}\right)=\left(-\mathbf{b}_{3}\right)$. This nonuniqueness brings about an interesting new feature of the dislocation network topology in BCC crystals not present in the conventional dislocation networks consisting solely of binary junctions. In the latter, it is possible to trace every single line with $1 / 2<111>$ Burgers vector through each $<001>$ junction it enters. It is even possible to uniquely deconstruct the entire network into individual $1 / 2<111>$ lines. However, the topology of dislocation networks containing 4-nodes is different in principle: it is now impossible to specify which of the four dislocations in a particular 4-node are the parents and which is the product, and to "trace" a given $1 / 2<111>$ line through the network. While it is still possible to deconstruct the network into constituent lines, there are a combinatorially large number of different ways to do this. Formation of multi-junctions results in the topological irreversibility or untraceability of dislocation networks.

While the DD simulations provide credible support for the existence of multijunctions, it is desirable to verify this finding by a discrete atomistic model that does not 
rely on the continuum theory of dislocations. Figure 2(a) shows the result of one such simulation in which three different dislocations (LHS of equation (1)) were introduced into a small fragment of the BCC single crystal and then allowed to relax the lattice distortions produced by the inserted dislocations (see the Methods section for details). In the relaxed configuration (Fig. 2(a)), two distinct 4-nodes are instantly recognizable, as is the junction dislocation with the $1 / 2$ [111] Burgers vector (RHS of equation (1)). Taken together, the DD and the atomistic simulations appear convincing - multi-junctions should exist. Yet for ultimate verification, we rely on transmission electron microscopy (TEM) of molybdenum single crystals. Figure 2(b-d) shows three different views of a single fragment of the dislocation network containing a binary junction and a 4-node. Four lines entering the 4-node are numbered from 1 to 4 . A unique TEM signature of the symmetric 4-node is that, in certain contrast conditions, one of four dislocations must appear out of contrast while the other three lines remain visible. Appearance of a symmetric 4-node in each of the four TEM frames shown in Fig. 2(c,d) is unmistakable. Similar 4-node dislocation configurations were also found in other regions of the TEM foil leading us to believe that their occurrence may not be rare.

As stated above, multi-junctions appear to hold dislocations together more tightly than binary junctions. It is difficult to exactly quantify this difference because stress required to unzip a given junction depends on multiple factors, including dislocation line lengths and orientations, direction of applied stress and exactly how the junction is incorporated in the dislocation network. To qualitatively assess the relative holding power of binary and multi-junctions, we performed a large series of DD simulations in which both binary and multi-junctions are formed in the same geometries and subjected to the same straining conditions (see Supplementary Discussion 1 for the detailed results). Depending on the Burgers vectors and line orientations, the dislocations may mutually repel or attract each other. In cases when they attract, the lines either zip 
junctions or stay crossed ${ }^{12}$. The crossed attractive configurations do not hold dislocations together appreciably and are destroyed by application of a small stress whereas junction configurations exhibit significant strength. The superior strength of multi-junctions compared to binary junctions manifests itself in several ways. First, the multi-junctions form and exhibit measurable strength over a wider range of initial line orientations than the binary junctions. Second, in the collision geometries where both binary and multijunctions zip, the latter require much higher stress to unzip and release the dislocations. Finally, whereas the binary junctions could be eventually unzipped under all tested line and stress orientations, the multi-junctions were found to be indestructible across a wide range of line orientations and stress states. In such cases, rather than unzip and release the lines to glide under increasing stress (Supplementary Video 3), the multi-junction yields by repetitively emitting concentric dislocation loops and returning to its zipped configuration (Supplementary Video 4) thus forming a new regenerative dislocation source of the Frank-Read type ${ }^{15}$. In contrast, under no stress state was a binary junction observed to act as a regenerative dislocation source.

An important question we now attempt to answer is whether multi-junctions play a role in strain hardening, i.e. when continued straining demands increasingly higher stress. The small scale DD simulations reveal the amazing strength of multi-junctions and their propensity to act as regenerative sources and imply that these dislocation tangles may play an important role in strain hardening, but alone are inconclusive. At the same time, TEM micrographs have demonstrated the existence of multi-junctions in BCC molybdenum and are suggestive but, taken post-mortem, days after the straining experiments, do not fully prove the relevance of multi-junctions to strain hardening. Large-scale DD simulations present a unique opportunity to observe the formation of multi-junctions during straining and to quantify their effects on strain hardening, via in situ computational experiments. 
Due to the extreme computational cost of DD simulations, it has not been feasible to simulate dislocation ensembles large enough to compute strain hardening directly from the underlying motion and interactions of many dislocation lines. Recently, we developed a new DD code ParaDiS (for Parallel Dislocation Simulator) specifically designed to take full advantage of massively-parallel supercomputers ${ }^{16,17}$. For the first time, ParaDiS enables direct simulations of large strains and strain hardening in statistically representative dislocation ensembles. Figure 3 shows the data obtained in a series of three DD simulations of single crystal molybdenum subjected to uniaxial compression along two different directions (see also Supplementary Figure 1). The simulation parameters were chosen to represent molybdenum single crystals at an elevated temperature of $450^{\circ} \mathrm{K}$. In accordance with experiments ${ }^{8}$ (see also Supplementary Figure 2), the simulation of uniaxial straining along [001] direction exhibits a high rate of strain hardening, as evidenced by a pronounced slope of the stress-strain curve beyond the initial yield, whereas there is virtually no hardening exhibited in the [011] case (Figure 3(a)). Since dislocations of at least three distinct Burgers vectors must be present to form multi-junctions, they are expected to form more frequently in the [001] straining simulation where all four distinct Burgers vectors of $1 / 2<111>$ type are active and not the [011] case where only two of the four are active. Observed both in simulations and in experiment, the strong contrast in the orientation dependence of strain hardening makes it tempting to assert that the difference is somehow related to multi-junctions. This assertion is further supported by the diminishing multiplication rate observed in the [011] case compared to the rapid and steady dislocation multiplication observed in the [001] case (Figure 3(b)) explained by the propensity of multi-junctions to act as regenerative sources.

To test further the assertion that multi-junctions strongly influence the orientation dependence of strain hardening, we now perform a computational experiment that is 
impossible to reproduce in the laboratory. Specifically, we repeat the same [001] straining simulation but start with a modified initial configuration in which the Burgers vectors $\mathbf{b}_{3}$ and $\mathbf{b}_{4}$ are converted into $\mathbf{b}_{1}$ and $\mathbf{b}_{2}$, respectively, such that the total density of dislocations remains initially unchanged. By "doctoring" the initial structure in such a way, we preclude any possibility of multi-junction formation during the course of this simulation. The resulting stress-strain and stress-density behaviours differ markedly from the full [001] straining simulation (with all four Burgers vectors included). Instead, the behaviour is similar to that observed in the [011] straining simulation (Fig. 3(a,b)). This observation further reinforces our assertion that the high hardening rate in straining along the high symmetry (e.g. [001]) directions is related to the formation of the multi-junctions (see also Supplementary Discussion 2).

To clarify how the multi-junctions affect strain hardening, we investigate the relationship between the evolving dislocation microstructure and the instantaneous flow stress. As shown in Figure 3(c), over the range of dislocation densities common to all three simulations, the flow stress appears to be determined by the total dislocation density alone, irrespective of the orientation of the tensile axis and the number of active Burgers vectors suggesting that the weaker but more numerous binary interactions predominantly define the overall plastic strength. At the same time, the frequency of binary collisions is determined by the dislocation density that increases much more rapidly in the high symmetry [001] straining due to proliferation of new dislocation sources, multi-junctions. As shown in Fig. 3(d), the topological composition of the dislocation microstructure differs significantly for different straining directions at the same level of total dislocation density (see also Supplementary Figure 3). While in the [011] straining simulation both the total dislocation density and the fraction of lines involved in the multi-junctions saturate, the [001] straining simulation with the higher fraction of multi-junction configurations continues to evolve to considerably higher total densities and higher 
fractions of lines involved in the multi-junctions. Taken together, these observations indicate that the number of active Burgers vectors affects the rate of formation of new dislocation sources (multi-junctions) leading to significant differences in the accumulation of dislocation density and, thus, in the strain hardening rates.

In situ visual observations reveal that, even in the [001] straining simulations, the binary junctions form much more frequently than the multi-junctions. In fact, for as long as dislocations move on different planes they must intersect making formation of binary junctions unavoidable. In contrast, multi-junctions form infrequently, mostly by attachment of a third line to an existing binary junction. At the same time, while the binary junctions are observed to dynamically unzip and reform elsewhere, the multijunctions once formed are observed to endure. As illustrated in the progression of snapshots in Figure 4 (see also Supplementary Video 5), zipping of new multi-junctions takes place preferentially near the existing ones gradually building up a sub-network of multi-junctions, a strong and mostly static backbone of the growing microstructure.

It remains to be seen what role multi-junctions play in the intricate dislocation patterns formed during straining of high-symmetry crystals ${ }^{18}$ : indications are that multijunctions can serve as strong anchors for dislocation tangles, braids, walls, etc. Another interesting question is whether multi-junctions form in crystals other than BCC: we predict that a variety of strong multi-junctions should exist in the face-centred-cubic and related high-symmetry crystals. Finally, theoretical analysis, DD simulations and TEM observations all suggest that dislocation tangles even more complex than ternary junctions exist, but they are rare and their stability is likely to be marginal. 
Methods

Dislocation Dynamics Simulations. In a DD simulation, dislocations are represented by piece-wise straight segments interacting with each other according to the equations of the continuum elasticity theory ${ }^{19,20}$. In a simulation, each dislocation segment moves with velocity $\mathbf{v}=\mathbf{M} \cdot \mathbf{f}$ proportional to the net force $\mathbf{f}$ exerted on the segment by external loads and all other dislocation segments (here $\mathbf{M}$ is the mobility tensor). A single simulation step includes: (1) calculating the forces acting on the segments, (2) advancing the segments to new positions according to their velocities, and (3) performing changes in the line topology (connectivity) when collisions or node instabilities are encountered. The force on a dislocation segment is calculated as the negative derivative of the system's energy with respect to the segment's position. The elastic constants and dislocation mobility function were chosen to capture the behaviour of $\mathrm{BCC}$ molybdenum above its athermal threshold $\left(\sim 450^{\circ} \mathrm{K}\right)$. The mobility was independent of the local line direction in the glide plane, i.e. the plane containing both line direction and Burgers vector. Mobility in the direction normal to the glide plane was a small fraction $\left(10^{-6}\right)$ of the glide mobility. Screw dislocations, whose line directions are parallel to their Burgers vectors, were free to glide in any plane containing their line direction. All small scale DD simulations were conducted as though the configurations were in an infinite medium, whereas the larger scale straining simulations were performed in a periodic cube $5 \mu$ on the side.

Analysis of Dislocation Network Topology. The entire network is comprised of nodes and links. A node is where three or more lines merge together, and a link is any line segment connecting two nodes of the network. For the present analysis, we label any 3node that bounds a regular binary junction as a "normal" or N-node. Likewise, any 4node formed by two dislocations crossing each other is also labelled as an N-node. All 
other nodes are regarded as "multi-nodes" or M-nodes, including the symmetric 4-nodes shown in Figure 1(e) as well 3-nodes produced by dissociation of the symmetric 4-nodes. Three types of links can be now defined with respect to the types of nodes they connect: NN links, NM links and MM links. Here, notation NM is used for any link that connects an N-node to an M-node. To compute the fraction of lines involved in the multijunctions shown in Figure 3(d), we summed the lengths of all MM links with half the lengths of all NM links and divided this sum by the total length of all links. The colour scheme used in Figure 4 is as follows: all MM links are shown in white whereas the colour of NM links is graded from white at the M-nodes to green at the N-nodes. All NN links, including binary junctions, are shown in green.

Atomistic Simulation. The simulation volume was a small cube-shaped block of a perfect BCC single crystal, $17 \mathrm{~nm}$ on each side. The initial geometry contained three dislocations with Burgers vectors $1 / 2[\overline{1} 11], 1 / 2[1 \overline{1} 1]$, and $1 / 2[11 \overline{1}]$ intersecting at the block centre. The atom positions inside the block were then relaxed to mechanical equilibrium using the conjugate gradient method and an interatomic interaction function for molybdenum ${ }^{21}$. The atoms on the block surfaces were fixed throughout the simulation. To visualise crystal defects, only the atoms inside the block with energies exceeding the ideal bulk value by $0.095 \mathrm{eV}$ are shown.

Experiment. The experiments involved three steps: (1) compression of a single crystal molybdenum specimen to $1 \%$ total strain along the [001] axis, (2) cutting and thinning the deformed specimen along the ( $\overline{1} 01)$ plane to obtain electron transparent foils, and (3) TEM observations using a set of reflection vectors $\mathbf{g}$ that can reveal multi-junctions. In the view shown in Figure $2(b)$ the zone axis $\mathbf{Z A} \approx[\overline{1} 01]$ and the diffraction vector $\mathbf{g}=$ [020] making all four dislocations entering the 4-node visible. The views in Figures 2(c) and $2(\mathrm{~d})$ were obtained using $\mathbf{g}=[\overline{1} 2 \overline{1}]$ and $\mathbf{g}=[121]$ which made lines $\mathbf{b}_{1}=1 / 2[111]$ and $\mathbf{b}_{2}=1 / 2[\overline{1} 1 \overline{1}]$ invisible due to $\mathbf{g} \mathbf{b}_{1}=0$ condition. To access additional diffraction 
vectors, the specimen was tilted to a new zone axis $(\mathbf{Z A} \approx[\overline{2} 01])$ making it possible to identify the Burgers vectors of two remaining dislocations in a similar manner: $\mathbf{b}_{3}=$ $1 / 2[11 \overline{1}]$ and $\mathbf{b}_{4}=1 / 2[\overline{1} 11]$, respectively.

\section{References}

1. Orowan, E. Zur kristallplastizitat. Zeitschrift Phys. 89, 605-659 (1934).

2. Taylor G. The mechanism of plastic deformation in crystal. Part I. Theoretical. Proc. Roy. Soc. A 145, 363-404 (1934).

3. Polanyi M. Uber eine Art Gutterstorung, die einen kristall plastich machen konnte. Zeitschrift Phys. 89, 660-664 (1934).

4. Hirsch, P. B., Horne, R. W., and Whelan, M. J. Direct observations of the arrangement and motion of dislocations in aluminium. Phil. Mag. 1, 677-684 (1956).

5. Hirth, J. P. and Lothe, J. Theory of Dislocations, $2^{\text {nd }}$ edn (Wiley, New York, 1982) pp. 9-13.

6. Saada, G. Sur ler durcissement dû à recombinaison des dislocations. Acta Metall. 8, 841-852 (1960). 
7. Franciosi, P., Berveiller, M. and Zaoui, A. Latent hardening in copper and aluminium single crystals. Acta Metall. 28, 273 -283 (1980).

8. Guiu, F. and Pratt, P. L. The effect of orientation on the yielding and flow of molybdenum single crystals. phys. stat. sol. 15, 539 - 552 (1966).

9. 5. Hirth, J. P. and Lothe, J. Theory of Dislocations, $2^{\text {nd }}$ edn (Wiley, New York, 1982) pp. 269-270.

10. Basinski, S. J. and Basinski, Z. S. in Dislocations in Solids, F.R. N. Nabarro, Ed. (North Holland, Amsterdam), vol. 4, pp. 261-362.

11. Nabarro, F. R. N., Basinski, Z. S., and Holt, D. B. The plasticity of pure single crystals. Advances in Physics 50, 193-233 (1964).

12. Madec, S. R. , Devincre, B. and Kubin, L. P. From dislocation junctions to forest hardening. Phys. Rev. Lett. 89, 255508 (2002).

13. Hähner, P. and Zaiser, M. Dislocation dynamics and work hardening of fractal dislocation cell structures. Materials Science and Engineering A 272, 443-454 (1999). 
14. Abraham, F. F. et al. Simulating materials failure by using up to one billion atoms and the world's fastest computer: Work hardening. Proc. Nat. Acad. Sci. 99, 5783-5787 (2002).

15. Frank, F. C. and Read, W. T. Multiplication processes for slow moving dislocations. Phys. Rev. 79, 722-723 (1950).

16. Bulatov, V.V. et al. Scalable line dynamics in ParaDiS. Supercomputing (2004). http://www.sc-conference.org/sc2004/schedule/pdfs/pap206.pdf

17. ParaDiS runs efficiently on two of the World's most powerful computers, Thunder and Blue Gene/L, both at the University of California Lawrence Livermore National Laboratory:

http://www.llnl.gov/computing/hpc/resources/OCF_resources.html\#thunder http://www.llnl.gov/computing/hpc/resources/OCF_resources.html\#bluegenel

18. Mughrabi, H. Dislocation wall and cell structures and long-range internal stresses in deformed metal crystals. Acta Metall. 31, 1367-1379 (1983).

19. Kubin, L.P. and Canova, G. R. The modelling of dislocation patterns. Scripta Metall. Mater. 27, 957-962 (1992). 
20. Ghoniem, N. M., Huang, J. M. and Wang, Z. Q. Affine covariant-contravariant vector forms for the elastic field of parametric dislocations in isotropic crystals. Phil. Mag. Lett. 82, 55-63 (2002).

21. Finnis, M. W. and Sinclair, J. S. A simple empirical N-body potential for transition metals. Philos. Mag. A50, 45-55 (1984).

Acknowledgements. We thank E. Chandler and C. Mailhiot for their encouragement and unwavering support of the ParaDiS code development effort, D. Lassila for providing the purified molybdenum crystals, M. LeBlanc for performing the mechanical tests, R. Cook for graphic design and F. Abraham and G. Campbell for critical reading and editorial suggestions for the manuscript. This work was performed under the Auspices of the U.S. Department of Energy by the University of California Lawrence Livermore National Laboratory under contract No. W-7405-Eng-48.

Correspondence and requests for materials should be addressed to V.V.B. (e-mail: bulatov1@1lnl.gov).

Supplementary Information accompanies the paper on Nature's website (http://www.nature.com). 

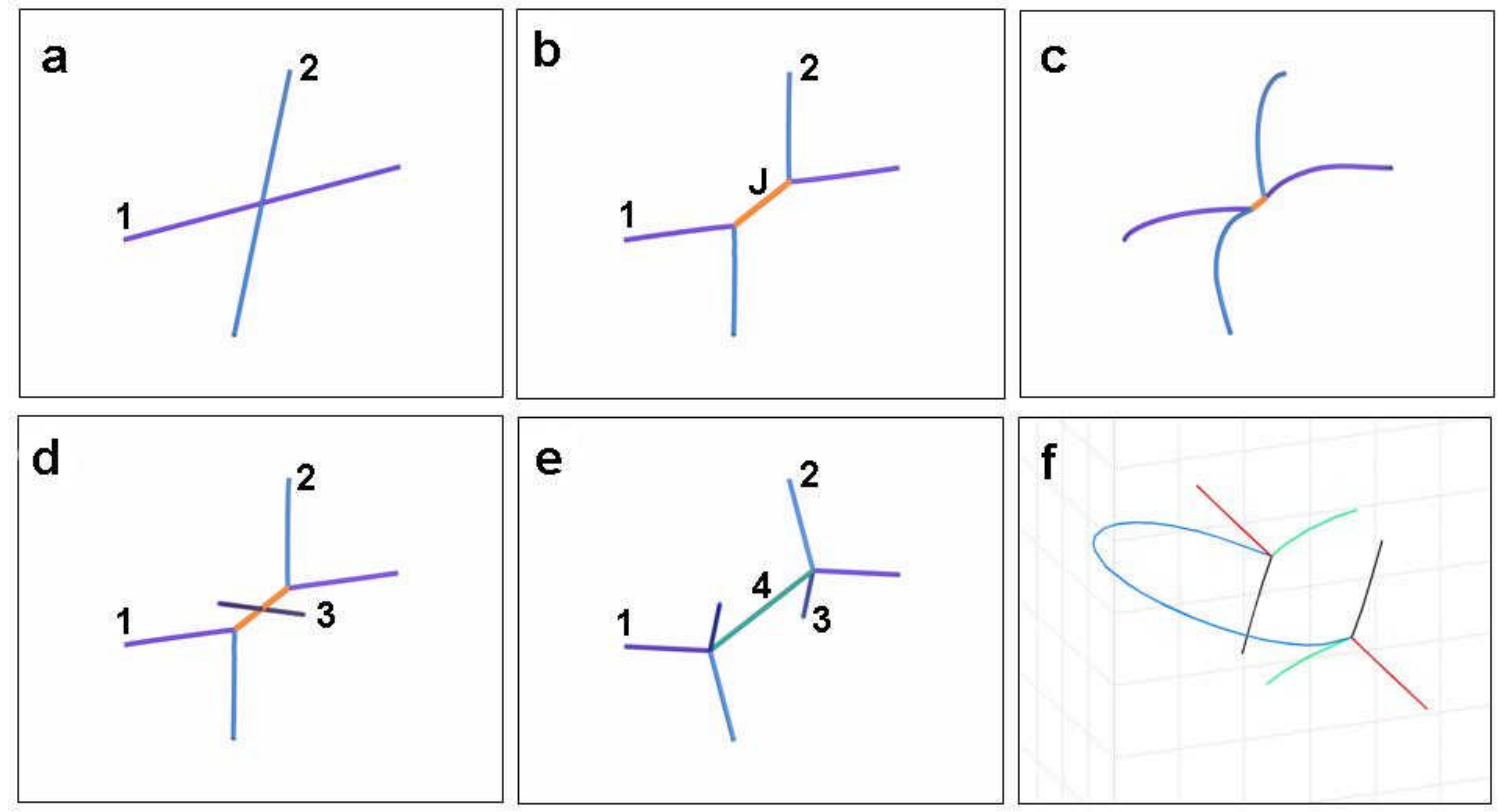

Figure 1: Formation (zipping) of dislocation junctions in the DD simulations. (a) Two dislocations lines are initially brought to intersection at their midpoints. (b) Once the interaction between two lines is turned on, two lines zip a binary junction. (c) A snapshot showing a binary junction unzipping under stress. (d) A third line is brought to intersect the binary junction. (e) The interaction among three lines makes them zip a long multi-junction. (f) A snapshot showing a multi-junction acting as a Frank-Read source of dislocation multiplication. 


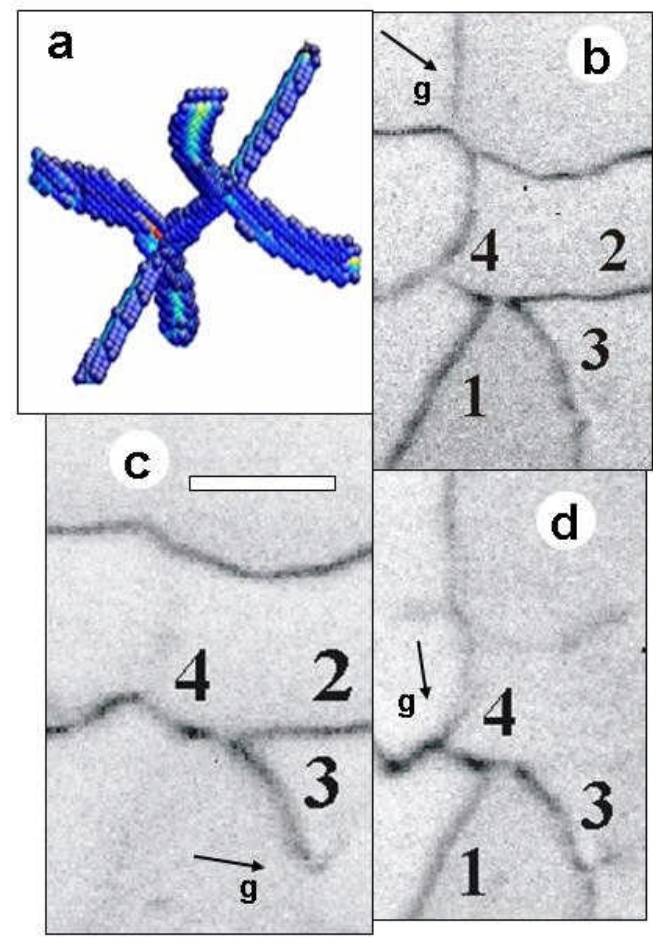

Figure 2: Atomistic simulations and experiments confirm that multijunctions exist in BCC metal molybdenum. (a) A multi-junction formed in an atomistic simulation. (b) A TEM micrograph containing a symmetric 4-node. In this view all four dislocations (1-4) entering the multi-node are visible. (c ) View in which dislocation 1 becomes invisible. The length of the scale bar is $0.2 \mu$. (d) Another view in which dislocation 2 is invisible. 

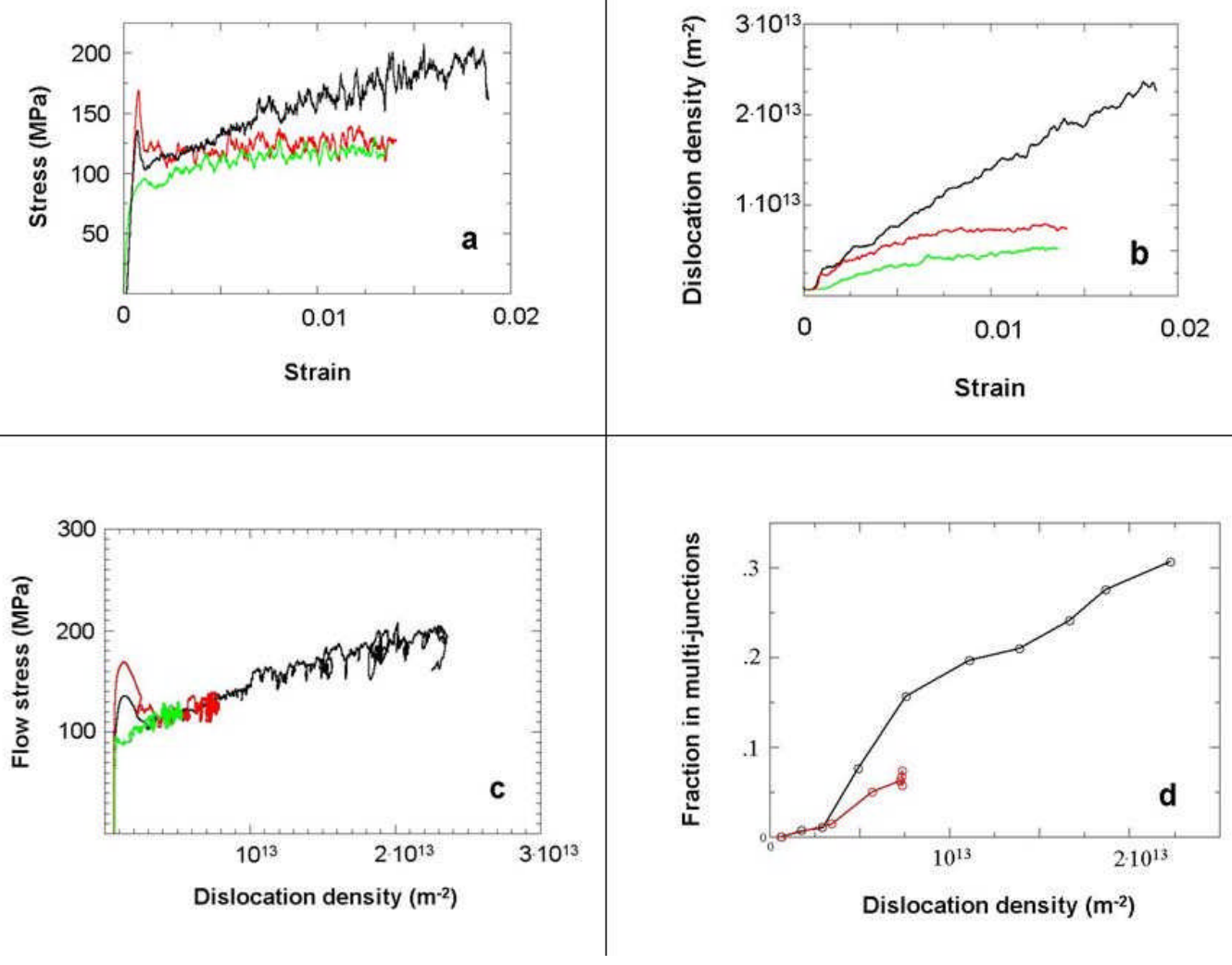

Figure 3: The results of virtual straining experiments on BCC molybdenum. The black lines correspond to the full [001] straining simulation, the red lines are for the [011] straining, and the green lines are for the "doctored" [001] straining simulation in which two of the four Burgers vectors are absent. (a) Flow stress as a function of strain. (b) Dislocation line density as a function of strain. (c) Flow stress versus total dislocation density. (d) The fraction of lines involved in multi-junction configurations as a function of the total line density. 


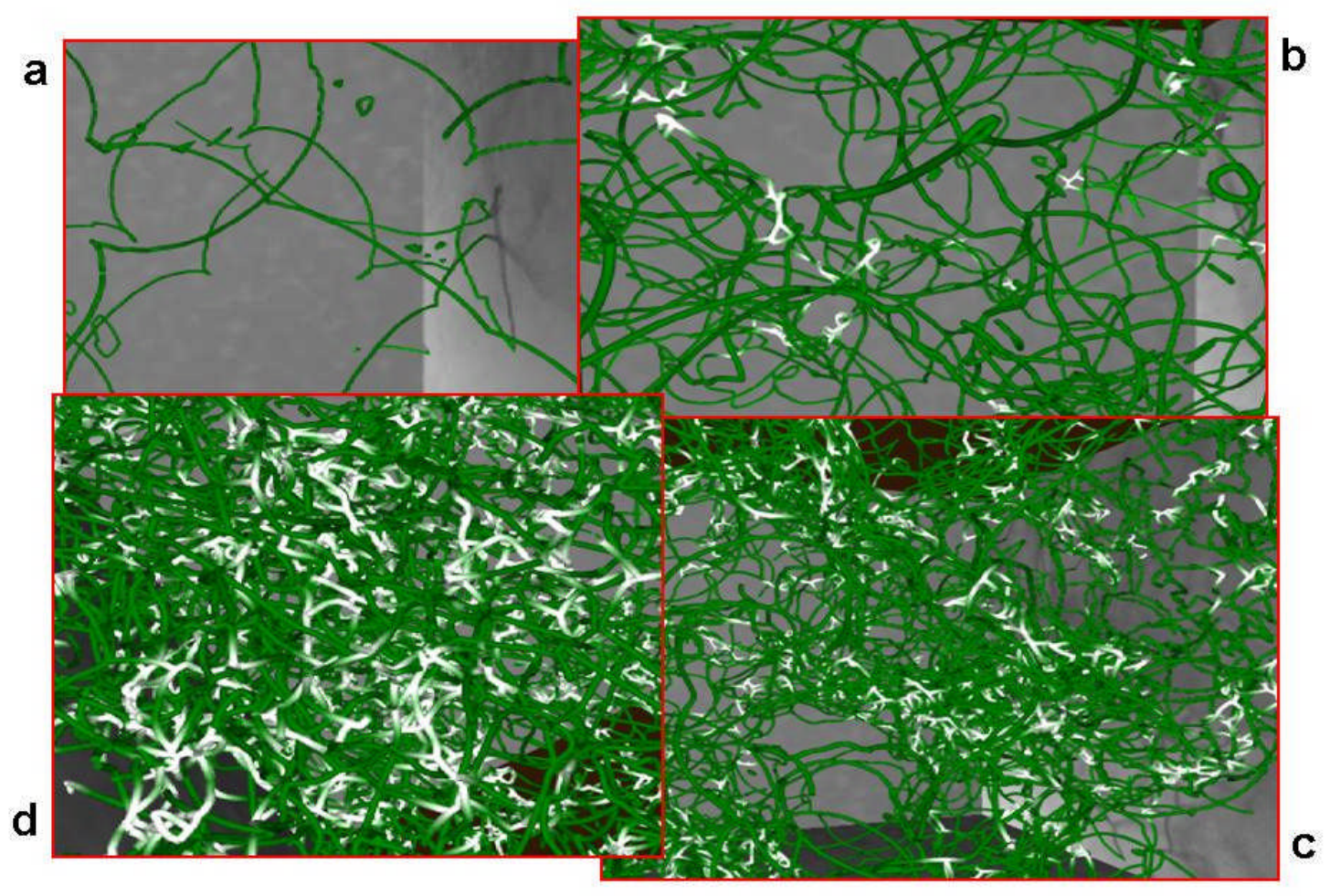

Figure 4: Snapshots of dislocation network evolution obtained from a DD simulation of [001] straining. (a) Initially, dislocation motion results in binary collisions only so that the network remains all green (see Methods section for a more detailed description of the colour scheme). (b) Near the yield strain $(\sim 0.2 \%)$ dislocations multiply and their collisions produce first few multi-junctions. (c) and (d) Continued dislocation multiplication results in increasingly frequent dislocation collisions leading to strain hardening and growth of the (white) subnetwork of multi-junctions. 